\title{
Analysis of left ventricular wall movement during isovolumic relaxation and its relation to coronary artery disease ${ }^{1}$
}

\author{
D. G. Gibson, T. A. Prewitt ${ }^{2}$, and D. J. Brown \\ From the Brompton Hospital, London
}

Left ventricular angiograms of 60 patients with ischaemic heart disease and 10 normal subjects were digitized frame by frame in order to study abnormalities of wall movement during the period of isovolumic relaxation. Plots were made of regional wall movement around the cavity throughout the cardiac cycle. In normal subjects 1.5 to $3.0 \mathrm{~mm}$ of symmetrical outward wall movement occurred during isovolumic relaxation, associated with an apparent increase of left ventricular volume of $10 \pm 4$ per cent. The corresponding peak velocities of wall movement were 4.3 to $5.7 \mathrm{~cm} / \mathrm{s}$, significantly less than those recorded in the same region of the cavity after mitral valve opening. In patients with ischaemic heart disease, the following abnormalities were encountered:

(1) Abnormal inward movement, which, in single coronary artery disease, occurred in the area supplied by the affected vessel.

(2) Abnormal outward movement of more than $6 \mathrm{~mm}$ in non-affected areas which appeared to be a compensatory phenomenon.

(3) An abnormal cavity shape change towards a more circular configuration before mitral valve opening.

(4) Reduced peak rates of wall movement in affected areas during systole and filling.

It is concluded that such inward wall movement during isovolumic relaxation is abnormal and a sign of local ischaemia whose presence has significant effects on overall left ventricular function in both systole and diastole.

Significant outward left ventricular wall movement has been shown to occur before mitral valve opening in normal subjects and in patients with prolapsing mitral valve cusp or ischaemic heart disease (Gooch et al., 1972; Altieri, Wilt, and Leighton, 1973; Ruttley et al., 1974; Hamby et al., 1974; Wilson et al., 1975). Such movements are usually reported as being localized to the antero-apical region though they may be accompanied by inward movement elsewhere around the left ventricular cavity border. However, there is still no general agreement as to their significance, since they have been considered a normal finding by some authors (Altieri et al., 1973) and as a manifestation of asynchronous relaxation by others (Hamby et al., 1974; Wilson et al., 1975). Part of this uncertainty may arise from Received 12 April 1976.

${ }^{1}$ Presented at 48th Scientific Sessions, American Heart Association, Anaheim, California, November 1975.

2Present address: Cooper Clinic, P.A., Fort Smith, Arkansas 72901, U.S.A. the technical difficulties in delineating a number of localized abnormalities occurring simultaneously around the border of the cavity and correlating them with measurements of overall ventricular functions such as changes in area or volume. This is particularly the case when angiograms are examined directly, frame-by-frame, and in order to reduce these difficulties, we have developed techniques for displaying information about local wall movement from single plane angiograms continuously throughout the cardiac cycle. Using these methods, it is possible to define abnormalities of left ventricular wall movement before mitral valve opening in greater detail than was previously possible, and to correlate them with overall ventricular function, and with the pattern of coronary artery involvement in patients with ischaemic heart disease.

\section{Subjects and methods}

Studies were made on 70 patients who were under- 
going left ventriculography and coronary arteriography for the investigation of chest pain thought to be the result of ischaemic heart disease. Of these, 60 proved to have coronary artery disease, and the remainder had normal haemodynamics, left ventriculograms, and coronary angiograms. This latter group of patients is referred to as 'normal', though it is recognized that they all suffered from chest pain whose aetiology was undetermined and that minor or hitherto undescribed abnormalities of left ventricular function might have been present.

The patients were studied supine, and were premedicated one hour before the start of the procedure with $200 \mathrm{mg}$ amylobarbitone sodium, given orally. Left ventriculography was performed in either the right anterior oblique or the posteroanterior position, and in 15 patients biplane posteroanterior and lateral films were obtained. Left ventriculography was always performed before coronary arteriography, using 35 to $40 \mathrm{ml}$ Triosil injected at a rate of up to $25 \mathrm{ml} / \mathrm{s}$. Cine film was exposed at 50 frames/s, and calibration was by means of a grid at midchest level. Multiple views of right and left coronary arteries were then obtained using Judkins' technique. Pressures were measured with a fluidfilled manometer system before the injection of any angiographic dye, and the zero level was the midthorax. Left ventricular end-diastolic pressure was taken as that before the start of the ' $a$ ' wave.

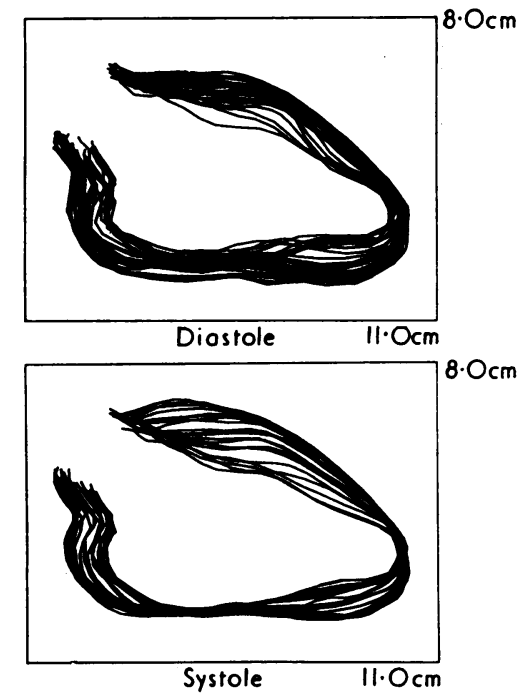

FIG. 1 Successive cavity outlines from the left ventriculogram of a patient with right and circumflex coronary disease. There is inferior akinesia and an area of abnormal movement on the anterior margin during diastole.

\section{Analysis of angiograms}

Angiograms were analysed as previously described (Gibson and Brown, 1975a), frame-by-frame, using a Summagraphics digitizer and a Prime $300 \mathrm{com}-$ puter system, and the information stored on disc for further calculations. Plots were made of superimposed cavity outlines during systole and diastole, a typical example being given in Fig. 1. Cavity area was calculated by numerical integration, and plotted throughout the cardiac cycle. Volume estimates were derived from area, and were used to calculate ejection fraction in the usual way. Left ventricular shape index was calculated as previously described (Gibson and Brown, 1975b) as $4 \pi$ Area/Perimeter ${ }^{2}$, which has a maximum value of 1 or 100 per cent when the cavity outline is circular, and which drops below this value when the outline is any other shape. Overall shape index changes throughout the cardiac cycle were noted, as well as those between the time of minimum area and mitral valve opening.

Plots of superimposed cavity outlines give only limited information about localized wall movement. In the angiogram illustrated in Fig. 1, it is apparent that the systolic frames show an area of inferior hypokinesia, but that the amplitude of wall movement in this area is normal during diastole. In addition, the diastolic frames also show a disturbance of wall movement involving the anterior

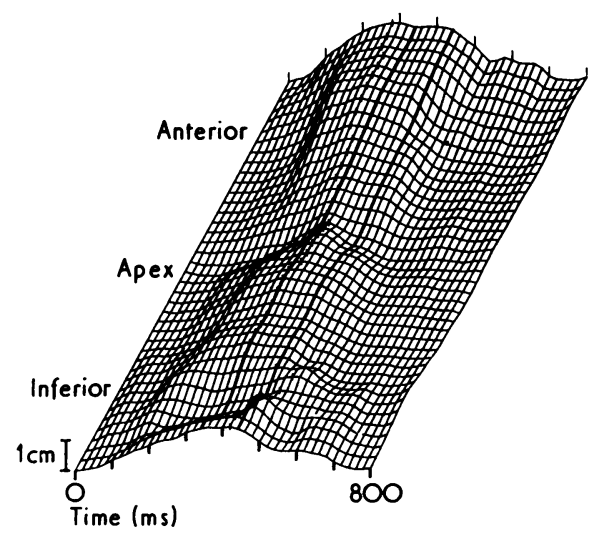

FIG. 2 Plots of regional wall movement against time from a normal left ventriculogram. Each horizontal plot represents wall movement at one site around the perimeter of the cavity. The diagonal lines are isochrones, connecting events occurring simultaneously. The two accentuated isochrones represent the times of minimum area and mitral valve opening. 
wall, but give no information as to its direction or timing. A more comprehensive method of display is, therefore, required, which shows wall movement continuously throughout the cardiac cycle, in all regions of the cavity.

The method adopted was based on multiple plots of wall movement against time around the cavity. Forty equally spaced points were defined on the cavity outline of the end-diastolic frame, starting from the border of the aortic root, adjacent

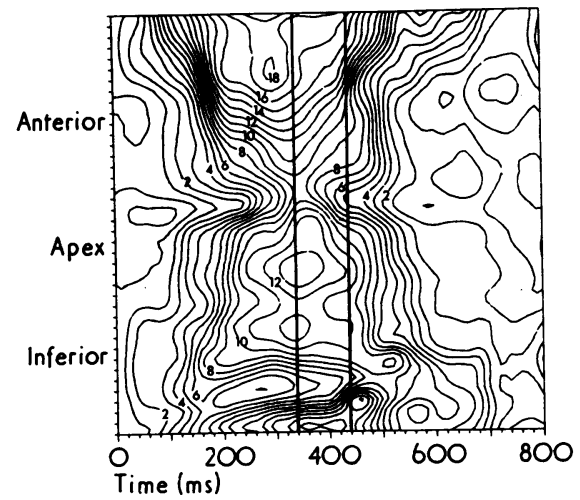

FIG. 3 Display of inward wall movement with position around the cavity, and time from the same data as Fig. 2. Inward wall movement is indicated by contour lines, which represent distances in $\mathrm{mm}$ shown by the figures, from the position of the wall in the frame with maximum cavity area. The vertical lines represent the times of minimum cavity area and mitral valve opening.

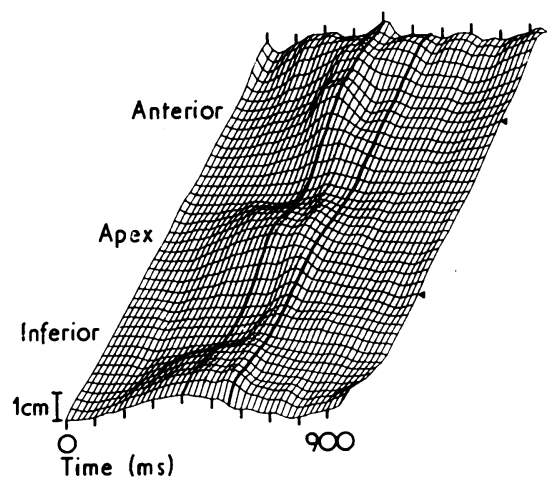

FIG. 4 Plots of wall movement from the data of Fig. 1, with layout as in Fig. 2. During isovolumic relaxation, outward wall movement along the anterior border, and inward movement along the inferior surface are apparent. The two plots marked by arrows are analysed in more detail in Fig. 8.

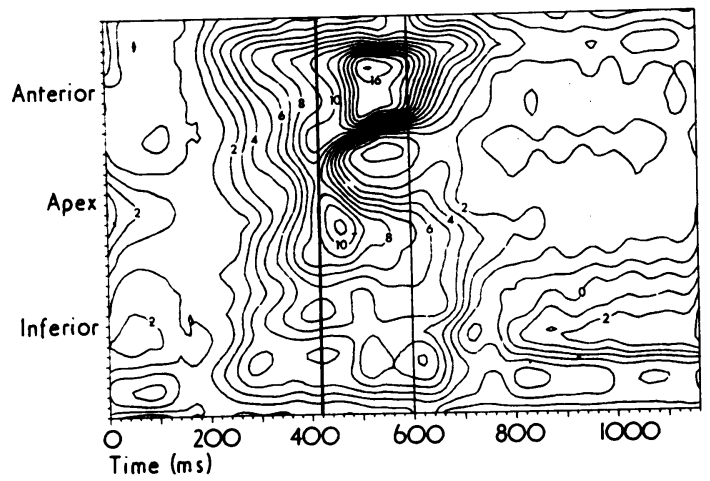

FIG. 5 Display of wall movement in a patient with triple coronary artery disease. The layout is the same as in Fig. 3. Abnormal wall movement during isovolumic relaxation is apparent. For full description, see text.

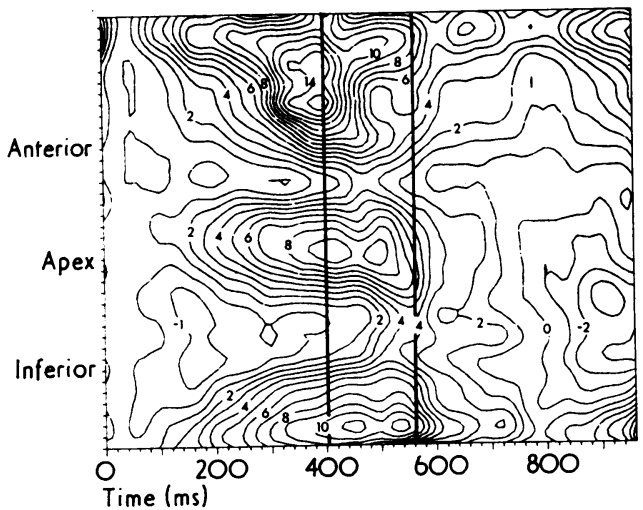

FIG. 6 Display of wall movement from the same patient whose data are shown in Fig. 1. For description, see text.

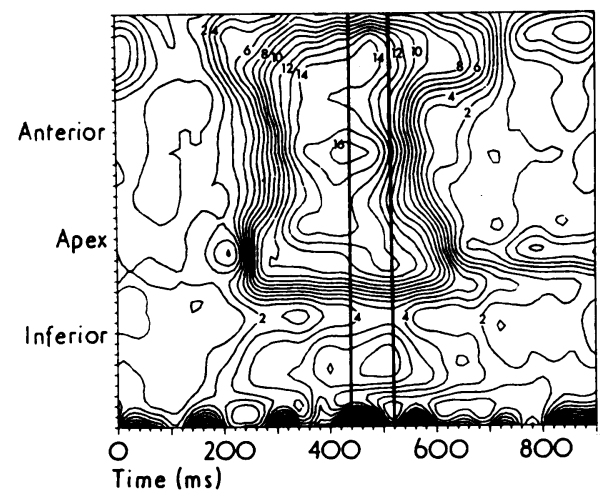

FIG. 7 Display of regional wall movement from a patient with an inferior aneurysm. Wall movement elsewhere is normal. For full description, see text. 
to the mitral valve, and proceeding anticlockwise. From each, the nearest point on the end-systolic outline was sought, and along the line thus defined, wall position in each cine frame was measured and expressed in $\mathrm{mm}$ from the end-diastolic position. Local wall movement could have been displayed in a variety of ways, but it was noted that, if plots of movement against time for each line were stacked obliquely, with a series of isochrones superimposed, representing the timing of successive cine frames, then a three-dimensional impression was created, from which localized abnormalities of movement of the cavity outline could readily be discerned. Absolute values were derived from a $1 \mathrm{~cm}$ calibration bar plotted alongside all records (Fig. 2 and 4) and inward wall movement shown as an upward or positive deflection. This was a versatile method of display, but suffered from the disadvantage that systolic events appeared foreshortened in a view that gave optimal representation of diastolic events. Though this could have been remedied by adopting a negative deflection for inward wall movement, this led to foreshortening of diastolic events.

An alternative display, based on the same data, is shown in Fig. 3 and 5 to 7. Here, inward movement of the wall from its diastolic position in the frame with maximum cavity area, is indicated by contour lines, each joining points which have undergone the same displacement, and successive contours representing inward or outward movement of $1 \mathrm{~mm}$.

Both types of display were interpreted in terms of events relating to the cavity as a whole. Isovolumic relaxation was taken as starting at the time of minimum cavity area and ending with mitral valve opening, which was identified as the timing of the frame in which unopacified blood from the left atrium first appeared within the left ventricular cavity. These two events were superimposed on all records.

\section{Analysis of records}

Left ventricular wall movement during isovolumic relaxation was assessed from the displays of cavity outline constructed as described above. For simplicity, the cavity was considered in three regions: anterior, apical, and inferior. For each of these, the plot of its outline showing greatest inward or outward displacement was identified, and overall amplitude and direction of movement during isovolumic relaxation were noted. For these selected regions, plots were also made of velocity of inward or outward movement as in Fig. 8.

Left ventriculograms and coronary arteriograms were reported on by a radiologist who was unaware of the results of regional studies of wall movement.

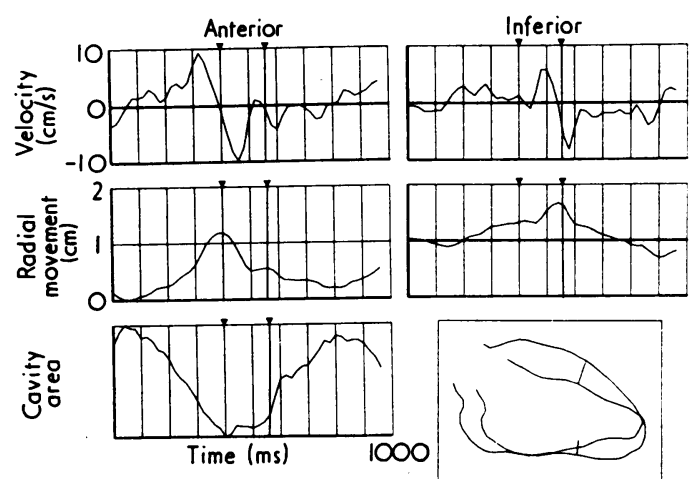

FIG. 8 Plots of local wall movement and velocity against time, from anterior and inferior regions of the angiogram shown in Fig. 1. From below, panels represent change in cavity area, wall position, and rate of change of position with time. The origin of the two traces are shown diagrammatically in the panel on the right, and also in Fig. 4. The times of minimum cavity area and mitral valve opening are indicated.

On the basis of this information, left ventricular contraction pattern was divided into one of the following groups: normal, segmental abnormality with normal ejection fraction ( $55 \%$ or greater), segmental abnormality with reduced ejection fraction, generalized impairment of function, and aneurysm, the last category being reserved for those patients in whom the diagnosis was confirmed at operation. Significant stenosis of a coronary artery was considered to be present if 70 per cent or more narrowing of the diameter of the lumen could be shown in any projection. No attempt was made to estimate collateral flow. The relation between abnormalities of wall movement during isovolumic relaxation and other manifestations of coronary artery disease was noted, including the pattern of coronary artery involvement, left ventricular contraction pattern, ejection fraction, and left ventricular end-diastolic pressure.

Standard t-tests were used to assess the significance of differences between means. 'Normal' values were taken as those lying within a range of 2 standard deviations of the corresponding means of the normal group.

\section{Critique of methods}

\section{(1) Digitization procedure}

This technique involves following the outer border of the image of the opacified left ventricular cavity with a cross-wire and identifying the co-ordinates of a relatively small number of points (about 100) 
on it. This procedure clearly contains a significant subjective element, particularly at end-systole when the cavity shape is irregular. In addition, it is not possible to follow minor irregularities of cavity outline using less than 100 points to define the cavity, so that an element of smoothing is introduced. However, as each outline is digitized separately, with no knowledge of the exact position of the previous one, reproducibility can be assessed from the relation of the border from frame to frame. Judged in these terms, it seems that the subjective component in the digitization procedure remains relatively constant throughout, but its absolute magnitude will only become apparent when comparison can be made with angiograms processed according to clearly defined algorithms.

\section{(2) Reference point for cavity outlines}

There is, at present, no general agreement as to the way in which successive cavity outlines should be related to one another. It seems to be widely assumed that for each cine frame, a single point should be regarded as fixed, but it is not clear which point should be chosen. A number have been proposed, including the aortic root (Rees et al., 1971; Helfant, Herman, and Gorlin, 1971), the intersection of major and minor axes (Hamilton, Murray, and Kennedy, 1972; Chatterjee et al., 1973), a line from the apex towards the aortic root, dividing the cavity area into two halves (Leighton, Wilt, and Lewis, 1974), or an external reference (Chaitman, Bristow, and Rahimtoola, 1973). It must be stressed that all these solutions are merely approaches to the analysis of the angiographic image of the left ventricular cavity, and are all equivalent to one another, provided that relative movement of the reference points is known. None can give information additional to that in the angiogram itself.

\section{(3) Physical significance of cavity outline}

The left ventricular cavity outline as displayed on an angiogram cannot be taken as representing the position of the corresponding region of left ventricular endocardium. Animal experiments have shown that the outer margin of the dye may be separated from metal markers on the myocardium itself, presumably by endothelial infolding, particularly at end-systole (Mitchell, Wildenthal, and Mullins, 1969). Outward movement of dye during early relaxation need not necessarily represent outward movement of endocardium, but could result from entry of dye-containing blood into the relaxing trabecular system. The irregular ventricular shape at end-systole may also result in the presence of concave, as well as convex, regions of wall, implying that the cavity boundary in a single projection need not necessarily represent continuous endocardium. In addition, if localized and rapid movements occur, different regions of wall may form the boundary at different times. The conclusion cannot be avoided that these factors represent definite limitations of the use of current angiographic techniques in studying wall movement at this time in the cardiac cycle.

\section{(4) Relation between wall movement and local function}

A major objective of defining left ventricular wall movement is to use the information to assess local function. The solutions outlined above for expressing wall movement have been devised and validated in studies of systolic function, and it is not certain that an identical reference point should be used in diastole. Further, the present study is concerned with regional abnormalities of wall movement, and it cannot be assumed that, in these circumstances, a single reference point is valid for the whole cavity, or even for the same region at different times in the cardiac cycle. We have, therefore, taken the simplest method, referring cavity outlines to a fixed point on the margin of the cine frame (Chaitman et al., 1973), and have attempted to define local movement in local terms by a method which allowed the direction in which it was measured to vary in different parts of the cavity in accordance with the orientation of the wall. Finally, we believe that the problem of the relation between wall movement and local function is a very complex one depending on such factors as ventricular architecture, local fibre orientation in different layers of the myocardium, and local constraints by other parts of the heart and extracardiac structures, and we do not feel that it can be solved by manipulation of reference points. As far as possible, therefore, other criteria have been used to corroborate conclusions about local ventricular function.

\section{(5) Use of single plane angiography}

The use of single plane angiography has potential disadvantages in demonstrating regional abnormalities of movement, since only those near the perimeter in any projection are likely to be detected. In order to gain some idea of the sensitivity of the method in this situation, a comparison was made in 11 patients in whom posteroanterior and lateral angiograms were available, at least one of the pair showing an abnormality of isovolumic relaxation. The two planes agreed in estimating outward movement as either normal or abnormal in 9/11 cases, and inward movement in 5/11 cases. The 
results indicate the value of biplane angiography in studies such as the present one, and give some indication of the frequency with which abnormalities may be missed if only one projection is used.

\section{Normal subjects}

In the 'normal' subjects, the mean interval between minimum cavity area and mitral valve opening was $93 \pm 13 \mathrm{~ms}$ (mean \pm 1 standard deviation) and, during this time, significant outward left ventricular wall movement occurred in all, leading to an apparent increase in volume equal to $10 \pm 4$ per cent that at end-diastole. This was caused by $3 \cdot 1 \pm 1.2$ $\mathrm{mm}$ outward wall movement in the anterior part of the cavity, $1.7 \pm 2.0 \mathrm{~mm}$ at the apex, and 1.5 \pm $2.5 \mathrm{~mm}$ along the inferior surface. In only 1 normal subject was inward movement observed in this period, of only $1 \mathrm{~mm}$ along the inferior surface. The peak velocity of outward wall movement during isovolumic relaxation was $5 \cdot 7 \pm 2.7 \mathrm{~cm} / \mathrm{s}$ along the anterior margin of the cavity compared with $12.5 \pm 2.8 \mathrm{~cm} / \mathrm{s}$ after mitral valve opening. The corresponding figures for the apex were 4.6 $\pm 4 \cdot 5$ and $9.0 \pm 3.0 \mathrm{~cm} / \mathrm{s}$, and for the inferior surface $4.3 \pm 3.4$ and $9.3 \pm 2.8 \mathrm{~cm} / \mathrm{s}$. In all normals the velocity of outward wall movement was greater after mitral valve opening in all three sites. The overall pattern of left ventricular contraction and relaxation can best be appreciated from the display in Fig. 2 and 3, which indicate the synchronous onset of contraction, relaxation, and wall movement during filling common to all the normal group.

Mean ejection fraction in the normal group was $72 \pm 7$ per cent, and the overall change in shape index in the cardiac cycle $28 \pm 7$ per cent, of which $6 \pm 2$ per cent occurred before mitral valve opening.

\section{Ischaemic heart disease}

Definition of abnormal wall movement Although abnormal wall movement during isovolumic relaxation might have been defined in a number of ways, the most consistent appeared to be on the basis of the 95 per cent confidence limits for the amplitude of wall movement established in normal subjects during isovolumic relaxation. The amplitude of wall movement was thus considered to be increased if it was greater than $6 \mathrm{~mm}$ in an outward direction at any site around the cardiac border. Any inward movement along the anterior margin or inward movement with an amplitude of $2 \mathrm{~mm}$ or more at the apex or along the inferior border was also considered to lie outside the normal range.

Frequency The overall frequency of abnormal wall
TABLE 1 Relation of relaxation to contraction pattern

\begin{tabular}{|c|c|c|c|}
\hline \multirow{2}{*}{$\begin{array}{l}\text { LV contraction } \\
\text { pattern }\end{array}$} & \multicolumn{2}{|c|}{ Abnormal outward movement } & \multirow[t]{2}{*}{ Total } \\
\hline & Absent & Present & \\
\hline \multirow{9}{*}{$\begin{array}{l}\text { Normal } \\
\text { Segmental abnormality } \\
\text { normal ejection fraction } \\
\text { Segmental abnormality lov } \\
\text { ejection fraction } \\
\text { Generalized impairment } \\
\text { Aneurysm } \\
\text { Total }\end{array}$} & 8 & 8 & 16 \\
\hline & 9 & 14 & 23 \\
\hline & & & \\
\hline & 11 & 2 & 13 \\
\hline & 5 & 0 & 5 \\
\hline & 2 & 1 & 3 \\
\hline & $3 \overline{5}$ & 25 & 60 \\
\hline & \multicolumn{2}{|c|}{ Abnormal inward movement } & \\
\hline & Absent & Present & \\
\hline Normal & 7 & 9 & 16 \\
\hline $\begin{array}{l}\text { Segmental abnormality } \\
\text { normal ejection fraction }\end{array}$ & 4 & 19 & 23 \\
\hline \multicolumn{4}{|l|}{$\begin{array}{l}\text { normal ejection rraction } 4 \\
\text { Segmental abnormality }\end{array}$} \\
\hline low ejection fraction & 1 & 12 & 13 \\
\hline Generalized impairment & 4 & 1 & 5 \\
\hline Aneurysm & 2 & 1 & 3 \\
\hline Total & 18 & 42 & 60 \\
\hline
\end{tabular}

movement during isovolumic relaxation is given in Table 1, where its relation to the pattern of left ventricular contraction is shown. Increased amplitude of outward movement occurred in 25 patients, and most commonly involved the anterior margin of the cavity. Its commonest association was with segmental abnormalities of contraction in patients in whom ejection fraction was normal. Abnormal inward movement occurred in 42 patients, most frequently involving the inferior surface of the heart, and was even more closely associated with segmental contraction abnormalities, regardless of ejection fraction. Of these 42 patients, 17 showed outward movement elsewhere in the cavity, while of the 25 showing abnormal outward movement, inward

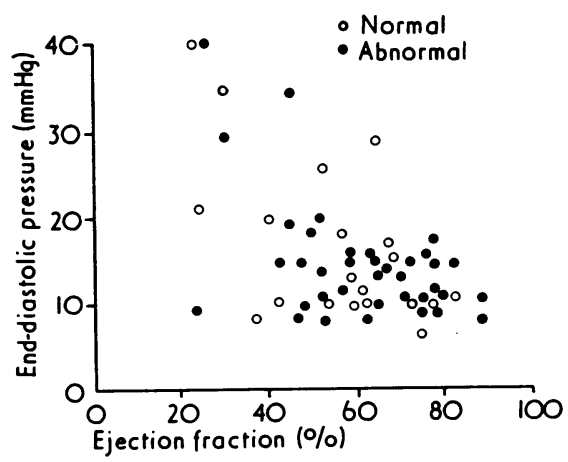

FIG. 9 Relation of abnormalities of relaxation to left ventricular end-diastolic pressure and ejection fraction. 
movement was present elsewhere in all but 6 . The relation between inward movement, ejection fraction, and end-diastolic pressure is shown in Fig. 9. In 12 patients only $(20 \%)$ was the amplitude of wall movement normal during isovolumic relaxation.

Site of abnormal movement The relation between the pattern of coronary artery involvement and the site of abnormal movement is given in Tables 2 and 3. Isolated disease of the right

TABLE 2 Relation between site of abnormal inward movement and coronary artery involvement

\begin{tabular}{lllcc}
\hline $\begin{array}{l}\text { Site of abnormal } \\
\text { inward movement }\end{array}$ & $L A D$ & $R C A$ & $\begin{array}{c}\text { LAD } \\
R C A\end{array}$ & Triple \\
\hline Anterior & $4^{\star}$ & 0 & 3 & 4 \\
Apex & 2 & 1 & 2 & 2 \\
Inferior & 0 & 5 & 9 & 10 \\
Total & 6 & 6 & 14 & 16
\end{tabular}

*1 patient with additional circumflex involvement.

LAD, left anterior descending; RCA, right coronary artery; Triple, 3-vessel disease.

TABLE 3 Relation between site of abnormal outward movement and coronary artery involvement

\begin{tabular}{llllll}
\hline $\begin{array}{l}\text { Site of abnormal } \\
\text { outward movement }\end{array}$ & LAD & $R C A$ & $C x$ & $\begin{array}{l}\text { LAD } \\
R C A\end{array}$ & Triple \\
\hline Anterior & 0 & $4 \dagger$ & 1 & 6 & 5 \\
Apex & 1 & 1 & 0 & 0 & 1 \\
Inferior & $4 *$ & 0 & 0 & 2 & 0 \\
Total & 5 & 5 & 1 & 8 & 6
\end{tabular}

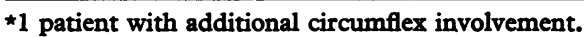
$\dagger 2$ patients with additional circumflex involvement.

coronary artery was associated with abnormal outward movement of the anterior surface, and isolated disease of the left anterior descending with that of the apex or inferior surface. When disease involved left anterior descending and right coronary artery or all three vessels abnormal outward movement was usually anterior, though any part of the cavity might be involved. The relation with coronary artery involvement was different for inward movement, in that isolated anterior descending disease was associated with inward movement along the anterior margin and isolated right coronary artery disease with abnormal inward movement along the inferior surface. With double or triple vessel disease inward movement was usually but not necessarily inferior.

Pattern of abnormal wall movement The peak rate of wall movement during isovolumic relaxation was $10.3 \pm 3.0 \mathrm{~cm} / \mathrm{s}$ in those regions showing ab- normal outward movement, which was significantly greater than normal $(P<0.001)$. In all but 5 patients, the velocity of outward movement during relaxation was greater than that during filling, the reverse of the normal pattern. In 8 patients peak velocity preceded mitral valve opening, while in the remainder (12) peak velocity of outward wall movement and mitral valve opening were synchronous. The mean velocity of outward wall movement during filling was $9 \cdot 0 \pm 4.4 \mathrm{~cm} / \mathrm{s}$, significantly less than normal $(P<0.01)$. The mean velocity of inward wall movement was $4.7 \pm 2 \cdot 2 \mathrm{~cm} / \mathrm{s}$ during isovolumic relaxation. The direction of wall movement reversed at the time of mitral valve opening, but during ventricular filling, the mean rate of outward movement was $6.8 \pm 4.0 \mathrm{~cm} / \mathrm{s}$, significantly less than normal $(P<0.001)$. No regional differences in peak rates of inward or outward isovolumic wall movement were apparent.

Systolic movement in affected areas The peak rate of inward systolic movement was normal in those regions that showed abnormal outward movement during isovolumic relaxation. However, regions showing inward movement during isovolumic relaxation showed a reduced rate of movement during systole. Very similar values were observed whether the anterior part of the ventricle was involved (peak systolic inward velocity $4.3 \pm$ $1.9 \mathrm{~cm} / \mathrm{s})$ or the inferior region $(3.2 \pm 1.6 \mathrm{~cm} / \mathrm{s})$. These were significantly less than normal $(P<0.001)$ and also less than unaffected regions of the same ventricle $(P<0.001)$.

Examples of disturbances of left ventricular wall movement occurring in patients with ischaemic heart disease are shown in Fig. 5 to 7. In the patient whose angiogram is represented in Fig. 5, the onset of inward wall movement is synchronous, though its velocity is reduced. During isovolumic relaxation, however, there is striking inward movement of the anterior border and outward movement of the apex, which has reached its diastolic position at the time of mitral valve opening. A more complex disturbance is illustrated in Fig. 6. The onset of inward wall movement is asynchronous, being delayed in the inferior part of the cavity, where amplitude and velocity of wall movement are also reduced. Inward wall movement involving the apex and inferior surface and outward movement anteriorly occur during isovolumic relaxation, and, finally, the velocity of wall movement during ventricular filling is reduced in all parts of the cavity. By contrast, the patient illustrated in Fig. 7 has an inferior aneurysm. Though the amplitude of wall movement is greatly reduced in this region, it is normal elsewhere throughout the cardiac cycle. 
Overall left ventricular function The presence of abnormal wall movement during isovolumic relaxation appeared to have significant effects on overall left ventricular function. The interval between the time of minimum area and mitral valve opening was increased in all patients with ischaemic heart disease, to a value of $120 \pm 18 \mathrm{~ms}$ when no isovolumic wall movement was apparent, and to $150 \pm 30 \mathrm{~ms}$ when abnormalities were present. These changes are significantly different from one another $(P<0.001)$, and also from normal $(P<0.001)$. Overall changes in left ventricular cavity shape were also abnormal in patients with ischaemic heart disease. The total reduction in shape index during systole was $16 \pm 3$ per cent in those patients with impaired isovolumic relaxation and $15 \pm 10$ per cent in those without, values not significantly different from one another. In order to allow comparison with the normal group, values were calculated for patients with ischaemic heart disease and normal ejection fraction. The corresponding figures were $18 \pm 6$ per cent for patients with abnormal relaxation and $16 \pm 6$ per cent for those without, both values being significantly less than normal $(P<0.001)$. However, when the change in cavity shape index occurring in the interval between minimum area and mitral valve opening is considered, patients with ischaemic heart disease and normal wall movement during this period showed a normal increase of $4 \pm 2$ per cent, while in patients with abnormal wall movement, but normal ejection fraction, it was $9 \pm 5$ per cent, or half the total change occurring in the cardiac cycle. The latter value was significantly greater than normal, expressed in absolute terms and also as a proportion of the total change in shape index occurring in the cardiac cycle $(\mathrm{P}<0.001)$.

\section{Discussion}

Isovolumic relaxation of the normal left ventricle is a complex process. Angiographic studies have shown that outward wall movement occurs while mitral and aortic valves are both closed, so that left ventricular cavity volume increases. There is no generally agreed explanation for this, though several mechanisms have been proposed, including herniation of the mitral valve apparatus or downward movement of the aortic valve cusps and the blood contained between them (Ruttley et al., 1974) into the left ventricle. In spite of the increase in cavity size, the period between the end of ejection and mitral valve opening is usually referred to as that of isovolumic relaxation, a convention we propose to follow.

Our results confirm previous ones in showing this outward wall movement (Altieri et al., 1973; Ruttley et al., 1974), though the method of display used enabled variation in its localization and extent to be more easily appreciated. The time of maximum inward movement at the end of systole was almost synchronous throughout the ventricle, and thus occurred simultaneously with minimum area. Outward wall movement before mitral valve opening was shown in all normal subjects, with no significant variation in its amplitude in different parts of the cavity. Inward movement occurred in only one subject, where it was confined to the inferior surface and its extent was small. The corresponding peak rates of wall movement were low and uniform in all regions of the ventricle. Cavity size increased slowly at first, but as the mitral valve opened, there was a sudden increase in its rate of change. This was reflected in local rates of outward wall movement which abruptly and uniformly increased, with significantly greater rates being recorded along the anterior margin than elsewhere. Isovolumic wall movement caused a significant change in cavity shape which became more circular. This was expressed quantitatively as a small increase in shape index of 6 per cent before mitral valve opening, compared with that of 22 per cent occurring during filling.

In the presence of ischaemic heart disease, this ordered process was frequently disturbed by two abnormalities of isovolumic relaxation occurring with approximately equal frequency: (1) excessive outward movement and (2) inward wall movement, the former being commoner along the anterior and the latter along the inferior margin of the cavity. Both types were often to be seen in the same cavity, and were probably complementary since, with mitral and aortic valves shut, excessive outward movement in one part of the cavity could only have been possible with inward movement elsewhere. In many patients, detection of only one abnormality was probably a limitation of single plane angiography, though when ejection fraction was low, failure to document outward wall movement as excessive reflected the use of criteria derived from normal subjects in patients in whom the amplitude of wall movement was reduced. These disturbances were frequently associated with segmental contraction abnormalities, and their distribution within the ventricle was characteristic in that the region of inward movement corresponded to that supplied by the involved coronary artery in patients with single vessel disease. In addition, these same regions showed delayed onset and significantly lower rates of inward movement during systole in comparison with other parts of the ventricle. We conclude that this pattern is the primary abnormality during 
isovolumic relaxation, and represents the behaviour of myocardium supplied by diseased coronary arteries. Conversely, excessive outward movement was confined to regions with normal systolic function and a normal coronary supply in patients with single vessel disease, strongly suggesting that it was a secondary phenomenon, a conclusion consistent with previous studies (Ruttley et al., 1974). The overall result of both was for left ventricular cavity shape to become significantly more circular before mitral valve opening, though the shape change during filling was significantly less.

Angiography was used in the present study to depict abnormalities of wall movement because it appeared to be the only one available to us capable of demonstrating rapid motion in different parts of the cavity. However, it has serious drawbacks, as has been pointed out. The first of these is the uncertain relation between the outer border of dye and the endocardium, particularly at end-systole. The same events were therefore studied by $M$-mode echocardiography, using a different physical principle to detect endocardium and record its motion throughout the cardiac cycle along with that of the anterior cusp of the mitral valve. The ability of this method to record related dimensional changes before mitral valve opening provides confirmatory evidence for their presence (Upton, Gibson, and Brown, 1976). A second problem inherent in any study which attempts to deduce regional abnormalities of function from wall motion is the extent to which the events described are local phenomena and the extent to which they result from overall movement of the heart within the thorax. The reasons for selecting the methods used in the present study have been discussed above, but it is possible, for example, that the apparent asymmetry of wall movement in normal subjects after mitral valve opening, with accentuation of that of the anterior wall, can be regarded as equivalent to slight overall movement of the heart upwards and to the left. By contrast, abnormal wall movements in patients with ischaemic heart disease appear to be truly local events, not only from their predictable relation to the pattern of coronary artery involvement, but also in view of the significantly greater velocities and consequently greater accelerations involved. If these local velocities are to be explained in terms of movement of the heart as a whole, the presence of large forces to produce the observed accelerations is implied, for which there is neither experimental evidence nor explanation.

Although it was not specifically designed to give information about basic mechanisms, the present study allows deductions to be made about the disturbances of function underlying the abnormalities of movement here described. Asymmetrical wall movement appears to be caused by regional ischaemia. It is closely associated with depressed systolic function, occurs in the territory supplied by diseased coronary arteries, and has been reported to regress after successful saphenous bypass grafting (Hamby et al., 1974). Depressed systolic function itself was not the primary cause since, in some patients, it occurred in the absence of any abnormality of relaxation (Fig. 7) while in others, maximum isovolumic inward wall movement was present in regions adjacent to those showing maximum depression of systolic function (Fig. 4). These abnormally relaxing areas seem to have reduced, but not absent systolic function, and so may represent transitional zones between normal and irreversibly damaged myocardium. Delayed relaxation of such an area would lead to tension persisting longer than elsewhere, and so to inward movement during early relaxation. Other possible explanations include myocardial distortion resulting from persistence of shear forces developed during systole or the direct effect of abnormal cavity shape at end-systole, since differences in regional tension depend on local curvature. Whatever the mechanism, it seems that observation of left ventricular wall movement during isovolumic relaxation can be used to give information about the physiological properties of the myocardium to supplement that obtained from recording systolic events.

Abnormalities of isovolumic relaxation may impair overall left ventricular function for a number of reasons. Outward wall movement, when ventricular pressure is still raised, is associated with loss of efficiency in energy transfer from myocardium to the circulation and, therefore, in depressed systolic function (Gibson and Brown, 1976). Prolongation of the period of relaxation, associated with a reduced peak rate of fall of ventricular pressure (McLaurin, Rolett, and Grossman, 1973) and delayed mitral valve opening, will impair filling at high heart rates, even if no other disturbance is present, simply by reducing the time available for inflow of blood. In addition, abnormal wall movement during isovolumic relaxation was associated with a reduced rate of outward movement after mitral valve opening and, therefore, with an abnormal filling pattern. We have previously suggested that part of the increase of ventricular volume during the rapid phase of filling may be accommodated by the cavity becoming more spherical (Gibson and Brown, 1975b). A change in cavity shape before mitral valve opening, and a reduced shape change during filling would interfere with this mechanism causing a reduction in ventricular compliance calculated from the ratio of 
pressure and volume changes without any alteration in the passive elastic stiffness of the myocardium itself. This effect may contribute to changes in compliance observed during angina or after trinitrin administration (Barry et al., 1974).

These fundamental disturbances caused by abnormalities of isovolumic relaxation in patients with ischaemic heart disease stress the remarkable nature of this process in normal man. It seems that an investigation of the events of early diastole can usefully be undertaken in any patient in whom overall left ventricular function appears to be impaired.

The computing equipment used in these studies was provided by the DHSS as part of their experimental computer programme.

\section{References}

Altieri, P. I., Wilt, S. M., and Leighton, R. F. (1973). Left ventricular wall motion during the isovolumic relaxation period. Circulation, 48, 499.

Barry, W. H., Brooker, J. Z., Alderman, E. L., and Harrison, D. C. (1974). Changes in diastolic stiffness and tone of the left ventricle during angina pectoris. Circulation, 49, 255.

Chaitman, B. R., Bristow, J. D., and Rahimtoola, S. H. (1973). Left ventricular wall motion assessed by using fixed external reference systems. Circulation, 48, 1043.

Chatteriee, K., Swan, H. J. C., Parmley, W. W., Sustaita, H., Marcus, H. S., and Matloff, J. (1973). Influence of direct myocardial revascularization on left ventricular asynergy in patients with coronary heart disease. Circulation, 47, 276.

Gibson, D. G., and Brown, D. J. (1975a). Measurement of peak rates of left ventricular wall movement in man. British Heart fournal, 37, 677.

Gibson, D. G., and Brown, D. J. (1975b). Continous assessment of left ventricular shape in man. British Heart fournal, 37, 904.

Gibson, D. G., and Brown, D. J. (1976). Assessment of left ventricular systolic function in man from simultaneous echocardiographic and pressure measurements. British Heart fournal, 38, 8.
Gooch, A. S., Vicencio, F., Maranhao, V., and Goldberg, H. (1972). Arrhythmias and left ventricular asynergy in the prolapsing mitral leaflet syndrome. American fournal of Cardiology, 29, 611.

Hamby, R. I., Aintablian, A., Tabrah, F., Reddy, K., and Wisoff, G. (1974). Late systolic bulging of left ventricle in patients with angina pectoris. Chest, 65, 169.

Hamilton, G. W., Murray, J. A., and Kennedy, J. W. (1972). Quantitative angiocardiography in ischemic heart disease; the spectrum of abnormal left ventricular function and the role of abnormally contracting segments. Circulation, 45, 1065.

Helfant, R. H., Herman, M. V., and Gorlin, R. (1971). Abnormalities of left ventricular contraction induced by beta adrenergic blockade. Circulation, 43, 641 .

Leighton, R. F., Wilt, S. M., and Lewis, R. P. (1974). Detection of hypokinesis by a quantitative analysis of left ventricular cineangiograms. Circulation, 50, 121.

McLaurin, L. P., Rolett, E. L., and Grossman, W. (1973). Impaired left ventricular relaxation during pacinginduced ischemia. American fournal of Cardiology, 32, 751 .

Mitchell, J. H., Wildenthal, K., and Mullins, C. B. (1969). Geometrical studies of the left ventricle, utilizing biplane cinefluorography. Federation Proceedings, 28, 1334.

Rees, G., Bristow, J. D., Kremkau, E. L., Green, G. S., Herr, R. H., Griswold, H. E., and Starr, A. (1971). Influence of aortocoronary bypass surgery on left ventricular performance. New England fournal of Medicine, 284, 1116.

Ruttley, M. S., Adams, D. F., Cohn, P. F., and Abrams, H. L. (1974). Shape and volume changes during 'isovolumetric relaxation' in normal and asynergic ventricles. Circulation, 50, 306.

Upton, M. T., Gibson, D. G., and Brown, D. J. (1976). Echocardiographic assessment of abnormal left ventricular relaxation in man. British Heart fournal, 38, 1001.

Wilson, C. S., Krueger, S., Forker, A. D., and Weaver, W. F. (1975). Correlation between segmental early relaxation of the left ventricular wall and coronary occlusion disease. American Heart Fournal, 89, 474.

Requests for reprints to Dr. D. G. Gibson, Cardiac Department, Brompton Hospital, Fulham Road, London SW3 6HP. 